\title{
How Computer Vision Provides Physical Retail with a Better View on Customers
}

\author{
Daniel Mora \\ AWS-Institute for Digitized \\ Products and Processes gGmbH \\ Saarbrücken, Germany \\ daniel.mora@aws-institut.de
}

\author{
Oliver Nalbach \\ AWS-Institute for Digitized \\ Products and Processes g GmbH \\ Saarbrücken, Germany \\ oliver.nalbach@aws-institut.de
}

\author{
Dirk Werth \\ AWS-Institute for Digitized \\ Products and Processes gGmbH \\ Saarbrücken, Germany \\ dirk.werth@aws-institut.de
}

\begin{abstract}
In recent years, web-based retailers have been taking over a growing market share from traditional brick and mortar retailers. One of the advantages leveraged by online retail is its ability to personalize the customer journey by analyzing the massive amounts of data that can be acquired easily in a digital environment. For example, click-streams from a web shop can help to identify a customer's interests in order to generate individual recommendations. To keep up, physical retailers, too, have to transform into the digital world. The main requirement for this are suitable data acquisition methods as well as resulting applications that are viable in an offline setting. In this paper, we investigate how recent technologies from the field of Computer Vision can overcome the data acquisition bottleneck and allow for data-based innovations that help traditional retailers to improve their customers' shopping experience and consequently to strengthen their market position. To this end, we introduce a conceptual tracking system for offline retail stores. Its purpose is to generate movement tracks over time for individual customers, from the time the supermarket is entered until it is left again. The acquired data allow for several data-based applications that can achieve similar goals as their counterparts in online retail.

Index Terms - retail, recommendations, computer vision, data acquisition
\end{abstract}

\section{INTRODUCTION}

Retail is a main pillar of the worldwide economy with a yearly sales volume amounting to about 25 trillion dollars [1]. While brick and mortar retailers, such as supermarkets or hardware stores, still constitute $90 \%$ of this number [2], e-commerce is growing tremendously fast, currently doubling its market share every four years [3]. This competition has been putting pressure on physical retailers and has already led to a wave of bankruptcies [4].

The success of online retailers, such as Amazon, Alibaba or Zalando, can be partly attributed to advantages enabled by the analysis of massive amounts of data about users and their shopping behavior which these companies can collect easily. One application is in recommender systems [5] which expose the customer to the right products at the right time, increasing convenience and likeliness of purchases [6]. A prime example is Amazon which dramatically increases its conversion rates by presenting a different, personalized digital store to each visitor [7]. Beyond the personal level, aggregated information about general shopping behavior also helps to continuously shape the overall structure and user interface of the shops.
To keep up with these developments, offline retailers need to transform [8]. Similar to online retail, the shopping experience has to become more appealing and engaging through personalization and interactivity [4]. Another key ingredient is omnichannel retail which tries to keep the customer connected at all times, providing information and recommendations through all available touch points [9].

The two main bottlenecks slowing down this transformation are the lack of means to address customers in real-time, while they are actually shopping in a store, and ways to acquire data from which to understand how the customer can be addressed and how the shopping experience can be enhanced in general. In particular, precise location-based data would open up a wide range of applications. Knowing which products a customer has been exposed to, in which order, when and for how long would enable applications like real-time recommendations and advertisements, better customer care and a better overall understanding of the situation inside a retail store (Sec. V).

Up to now, research has tried to tackle the aforementioned problems by using different technologies that share some disadvantages (Sec. II-B): First, they require a certain level of active cooperation by the customer but people are generally reluctant to cooperate without tangible incentive. Second, most existing technologies use specialized and potentially expensive hardware but only provide imprecise data.

In this paper, we choose a different approach and propose a visual data acquisition system for offline retail based on people tracking using conventional surveillance cameras (Sec. IV). As the input to the system is merely video data from those cameras, suitable hardware already exists in most retail environments. Using the system, the location of individual customers within a retail store can be tracked in real-time which yields a large set of movement paths corresponding to individual shopping sessions. Based on this type of data, a lot of promising applications (Sec. V) can be provided to store owners and customers alike, including: real-time dashboards for store managers, display-based and personalized recommendations, more efficient deployment of staff, shoplifting prevention and general store optimization. We also address the challenge of adhering to data protection laws such as the European Union's General Data Protection Regulation (GDPR) by designing the method to use and store as little data as possible, not relying on 


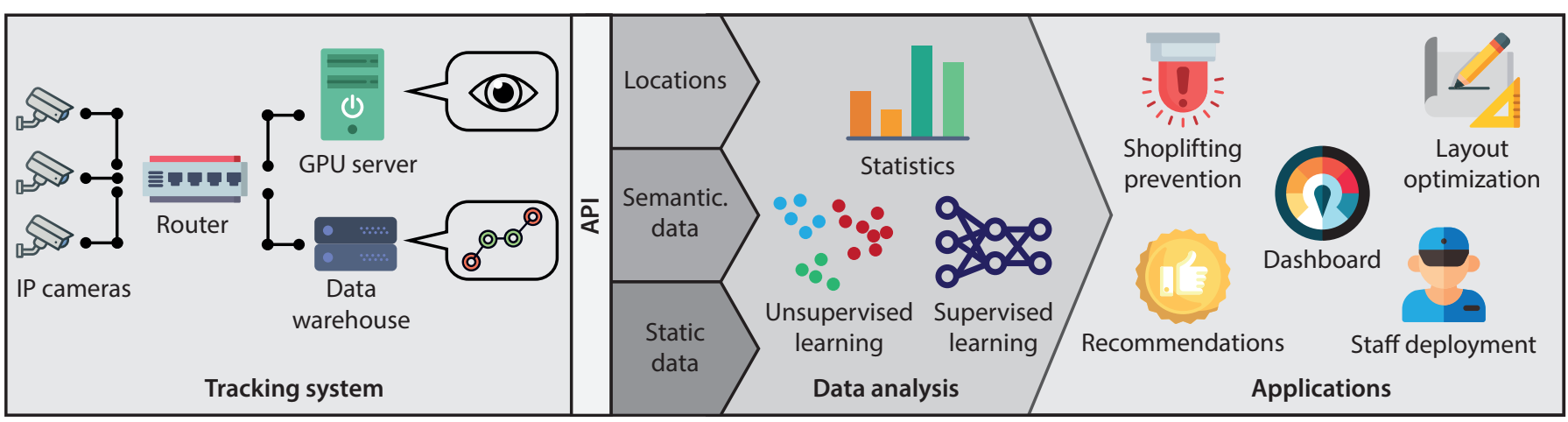

Fig. 1. We propose a visual acquisition system to acquire real-time movement tracks of individual customers in a physical retail store. Using methods from data analytics and machine learning, this enables a wide range of potential application that can help to narrow the current gap between online and offline retail.

connecting movement data in stores with personal information about the customer.

After discussing the gap between online and offline retail and previous approaches to offline data acquisition, in the next section, we give a brief overview of the technology behind visual tracking in Sec. III, in order to provide the context necessary to understand the subsequent proposal. Finally, we introduce our acquisition system in Sec. IV and list potential applications in Sec. V.

\section{PREVIOUS WORK}

To motivate our advocacy for visual tracking, we first investigate the differences between online and offline retail when it comes to data analysis (Sec. II-A). Taking a look at previous approaches to location-based data acquisition for brick and mortar retail (Sec. II-B) and drawing inspiration from related attempts of visual acquisition (Sec. II-C), we can identify the current limitations and eventually devise our own acquisition system (Sec. IV).

\section{A. The Gap Between Online and Offline Retail}

A drastic advantage of e-commerce is its ability to provide customers with customized real-time recommendations which reduce the number of active steps for a customer to reach a particular product. This includes identifying which products a customer is already looking for [10] but also making him or her aware of additional products which he or she does not have interest in yet but will likely have when confronted with them [11]. Such recommendations are made possible by the collection of explicit data, e.g., ratings and clicks, as well as of implicit data: If the customer has searched for a particular (type of) product, it is likely there is an interest in it. Another advantage of online retail is the possibility to adjust the design and structure of web shops effortlessly [12]. Data on how a website is used can be can logged and help to optimize it. The same is not as easily possible for traditional retail as it is very costly and time consuming to acquire similar data. Finally, while shoplifting is a significant problem in brick and mortar and amounts to a financial damage in the order of a hundred billion dollars [13], it does not exist in the same way for online retailers.

\section{B. Customer Tracking in Offline Retail and its Applications}

The idea of personalizing the shopping experience utilizing information technology is not an invention of e-commerce: In 1994, the year Amazon was founded, Asthana et al. [14] presented the idea of personalized shopping assistance devices, which integrated location data, speech recognition and other sources of data to provide real-time recommendations and engaged the customer in a personal conversation. Two decades later, many of the ideas of Asthana et al. have become reality in similar ways for online retail while they still appear futuristic for traditional retailers.

Even if other barriers blocking the realization of these ideas, such as expensive hardware and limited capabilities of artificial intelligence, have fallen, the access to data remains a challenge. To acquire detailed information about the behavior and movements of customers in retail environments, researchers have proposed a range of technologies. The most prominent ones are based on the Global Positioning System (GPS), radiofrequency identification (RFID) tags, Wi-Fi signal strength and (smart) beacons. We will now review their individual advantages, limitations and previous applications.

1) Wi-Fi RSS: Leveraging the Wi-Fi received signal strength (RSS) is currently the most popular way to infer location information for customers. This method exploits the relation between the location of mobile devices such as smartphones and the corresponding signal strength measured by nearby WiFi access points. The location of a device can be determined by combining information from multiple access points, mapping signal strength configurations to specific positions.

The first paper to use location-based data acquired via $\mathrm{Wi}-\mathrm{Fi}$ RSS for a recommender system was the one by Fang et al. [15] who compute the locations of customers in inside environments at store-level accuracy. Based on the visited stores, an accompanying mobile app provides contextual information and recommendations like products from specific categories or of particular brands to customers. Similar approaches are taken in [16] and [17], performing case studies in one and two shopping centers, respectively. While Chen et al. [17] also pursue the recommendation of stores, Zheng et al. [16] extend the set of possible applications by recommending indoor points 
of interest considering a user's preferences.

Despite its popularity, Wi-Fi RSS has two shortcomings First, it fully depends on the willingness of a customer to connect to a particular Wi-Fi network provided by the store, often even using a particular mobile application. Otherwise, no information can be acquired [17]. Second, the method is spatially inaccurate and only provides store-level accuracy [15] which limits the potential applications. Apart from the distance of a device to an access point, several other factors influence the received signal strength, too, for example objects blocking the line of sight between a device and the Wi-Fi hardware. This includes non-stationary "objects" such as other customers which means that many influences are not known in advance and therefore cannot be accounted for. These drawbacks are mitigated by visual tracking (Sec. IV): It can reach shelf-level accuracy and does not require active participation of the person being tracked.

2) RFID: Radio-frequency identification is using modulation of electromagnetic fields to transmit data [18]. Small tags storing a unique identifier, and potentially additional information, are attached to objects to make them identifiable and trackable.

While very popular for other applications related to retail, e.g., stock management and size-level replenishment [19], RFID-based tracking has not received as much attention yet One of the few examples is the paper by So and Yada [20] who equipped shopping carts in a grocery store with RFID tags to record their locations in the store over time, for complete shopping sessions. The improved accuracy of the tracking compared to Wi-Fi RSS allows for interesting applications: Departments in a store that are likely of interest to a user can be recommended via a mobile application. By analyzing statistics such as the time spent in a particular area, additional conclusions about the future shopping behavior can be drawn.

We can identify two limitations of RFID as a technology to acquire precise location data: First, additional hardware is required - RFID tags for each tracked object, antennas and RFID readers. Second, tracking is tied to the objects carrying the RFID tags, such as shopping carts in the case of [20], and their movements are not identical to those of the customers using them. Often, people leave their shopping carts in a larger aisle to search for products in more narrow aisles. In such cases, a lot of information on the customer's behavior is lost. Visual tracking does not suffer from these problems as it is using existing hardware and always follows the actual customer.

3) GPS: The Global Positioning System is the most popular satellite-based navigation system. Besides standalone GPS receivers, most mobile devices like smartphones, watches or cameras have the capability to use GPS-based positioning.

One of the few methods using GPS is the recent RecStore framework [21]. It is based on the Android Location API, that in turn is mainly based on GPS, and determines the locations of customers in shopping malls to provides storelevel recommendations according to a customer's preferences by merging information about previous purchases with location information. However, even if GPS is popular for outdoor positioning and readily available in mobile hardware, it often shows poor performance inside of buildings where most traditional retailers are located. Therefore, GPS is not an ideal technology to support the applications targeted in this paper.

4) Bluetooth Beacons: A final option to acquire locationbased customer data is offered by (Bluetooth) beacons. Such beacons are miniature computers equipped with a Bluetooth chip that are able to broadcast data packages. Localization using beacons works similar to Wi-Fi RSS (Sec. II-B1), taking a role analogous to that of $\mathrm{Wi}-\mathrm{Fi}$ access points, but may achieve a higher accuracy of a few meters [22] depending on the number of beacons and their placement.

Lacic et al. [23] suggest to use beacons distributed in public spaces such as shopping centers to track the locations of visitors. Based on the similarity of users regarding their location data, recommendations for relevant places to visit are given. However, the approach is only evaluated in theory. No actual beacon hardware is used and there are few applications. A similar approach is taken by Christodoulou et al. [24] who use Apple's iBeacon hardware to locate customers and push personalized notifications for which information provided by the user, such as a shopping list, is combined with data from tracking.

While beacons offer a sufficient accuracy to perform a tracking at the scale of individual shelves, due to their similarity to Wi-Fi RSS they share the limitation that active participation by the customer is required. Furthermore, the problem of generating full movement tracks, which are the foundation for the applications proposed in this paper (Sec. V), has not been addressed yet for the case of beacons.

\section{Visual Analysis in Retail}

One of the first papers discussing the use of video analytics in retail was the one by Neman et al. [25]. Using digitized recordings from surveillance cameras in a store an automated customer counting and tracking is performed. Only a single camera is used at a time and the applied tracking algorithm is, while easy to implement, computationally inefficient. The proposed method is therefore not suitable for real-time analysis of whole customer journeys, which is the goal of this paper. Additionally, the analyses of Neman et al. are mostly concerned with applications of customer counting, not tracking. A similar approach is taken by the authors of [26] who perform tracking of people in a supermarket for individual cameras, too. Their main use case is prevention of fraud by customers and cashiers but basic analyses such as customer counting and traffic flow are demonstrated as well. Due to the fact that the tracking is executed on a per-camera basis and information from multiple cameras is not merged to movement paths corresponding to full shopping sessions, the presented system represents a step forward but does not provide a sufficient source of data for the applications discussed in this paper (Sec. V).

A different use case from ours which has received some attention is the analysis of a customer's interaction with shelves and products. Frontoni et al. [27] try to classify activities performed by customers in front of shelves using RGBD images as input data. This allows to analyze the customers' reactions to changes in the store layout or product presentation. 
Also Liu et al. [28] use data from surveillance cameras to track shelf interaction. Their use case is the identification of situations of interest to a shop manager. For example, instances of customers picking up items but returning them to the shelf afterwards can be identified. Another emerging technology is eye-tracking. Using special eye-tracking glasses, worn by customers, their attention can be directly measured during the shopping experience [29]. Retailers can benefit from this technology as it discloses the visual impact of products, marketing campaigns or of the store environment, allowing them to better understand customer behavior and to take actions to stimulate customer engagement [30]. While the aforementioned approaches achieve a detailed (spatially) local analysis of customer behavior, many applications such as real-time dashboards or shoplifting prevention require a deeper understanding on a global scale and not some still rely on specialized hardware or activate participation of the customer.

Finally, a combination of video data with other means of tracking has been proposed by Sturari et al. [31] who combined beacons (Sec. II-B4) with an RGBD camera. While this combination improves upon both individual methods, the single camera used has a small field of view and only covers about $10 \%$ of the full shopping area. An extension to multiple cameras is not discussed which reduces its advantage over purely beacon-based methods for continuous tracking.

\section{BACKGROUND: VISUAL TRACKING}

To devise a visual tracking system that produces stable, realtime movement tracks for individual customers in a retail store, it is necessary to understand the basic algorithmic steps which are usually used to implement video-based tracking. We will now briefly review these steps on an abstract level and point out a few important realizations.

Visual tracking of people is a long-standing problem in Computer Vision research with many applications in fields such as security, robotics or safety. Some bear similarities to the situation addressed in this paper. For example, people tracking can be used to gain insights into the behavior and performance of athletes in soccer and other team sports [32] or counting of people [33].

Often, the structure of a tracking method is as follows: The input consists of video streams, potentially from multiple cameras. The output, for the case considered in this paper, are movement paths for individual persons, defined with respect to three-dimensional space over time. Mapping input to output is typically split into three algorithmic building blocks as shown in Fig. 2. First, a detection method that identifies regions in an image which likely contain a person (Fig. 2, left) and returns them, typically in the form of bounding boxes. Second, a tracking method that follows the detected persons over time by matching observations between consecutive frames (Fig. 2, middle) - in technical terms, only this computational step is called "tracking". Third, a method that translates the tracked positions, which are initially defined with respect to the camera image, to the originating three-dimensional positions. In our scenario, these positions can be further mapped to positions on the two-dimensional shop floor plane (Fig. 2, right).

\section{A. People Detection}

Object detection in videos is based on recognition of distinct structures. In addition to spatial structures, which can be detected in individual video frames, detection in videos can also exploit temporal structures by analyzing how the same pixel locations change over time. As identified in the recent survey by Benenson et al. [34] which compares more than 40 pedestrian detection methods, top-performing approaches often combine spatial and temporal cues.

Older methods for (spatial) people detection mainly use a two-step approach in which the input image is first transformed to a different representation by computing features like histograms of oriented gradients (HOG) [35], which describe local distributions of edge orientations, or wavelets [36] which capture frequency distributions in different parts of an image. This significantly lower dimensional representation then serves as input to a general classification method, e.g., support vector machines (SVMs) [37], which decides whether a certain combination of feature values corresponds to a person depicted in the image or not. Recently, also end-to-end approaches which replace the combination of pre-defined features and lowcomplexity classifiers by a single, complex (deep) artificial neural network [38] have received a lot of attention [39], [40]. This family of approaches drops the need to manually come up with descriptive features by autonomously learning the relevant features from examples. Another reason for the popularity of Deep Learning-based methods is their easy deployment using frameworks such as TensorFlow [41].

Regarding methods integrating temporal knowledge to better detect (moving) objects, given suitable conditions such as a mostly static background, background modeling has to be mentioned: By determining how the environment in which people are moving looks and capturing this using techniques such as Gaussian mixture models [42] the moving foreground objects can be separated more easily from the background.

\section{B. Object Tracking}

The tracking step establishes connections between the initially unrelated detections from several consecutive video frames. Again, a huge number of methods exists [43].

Tracking is frequently split into two sub-problems. On one hand, a distance measure between detections is defined to determine how likely they correspond to the same object. On the other hand, via optimization, a good assignment of pairs of detections from two frames is derived. Robust distance measures combine different cues, e.g., the overlap of two detections' bounding boxes can be combined with the distance between the appearance-based descriptors computed for the bounded regions, for example HOG or color histograms [44]. Finding the optimal assignment is a general mathematical problem which can be solved by algorithms such as the KuhnMunkres algorithm (or "Hungarian method") [45]. 


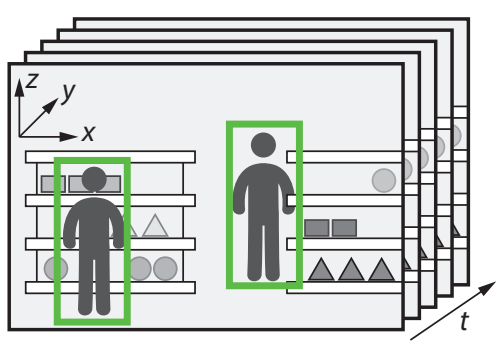

Object detection
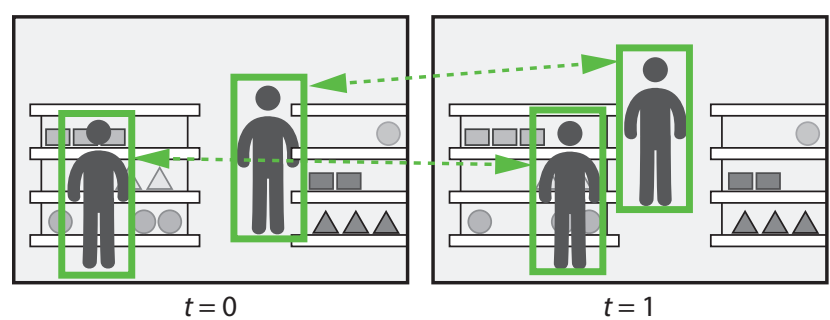

Tracking

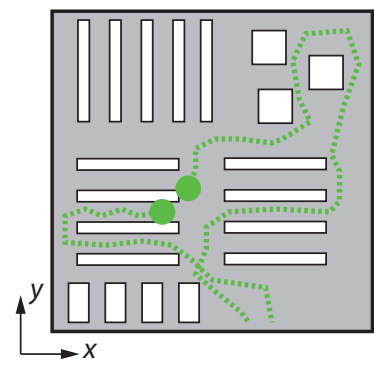

Position estimation

Fig. 2. Video tracking can be subdivided into three major steps: Detection of relevant objects (left), re-identifying them over time - the actual tracking (middle) - and estimation of the precise three-dimensional position of the tracked objects (right).

More advanced tracking methods extend per-frame descriptors to full object models which try to capture the appearance variations of the tracked objects over time [46] and possibly also their movement patterns [47] which is particularly useful if several objects show a similar visual appearance.

\section{Depth Estimation / Un-projection}

Tracking methods themselves only determine the positions of objects within the two-dimensional video frames (cf. Fig. 2, middle). However, we eventually have to determine the position of customers with respect to the floor plan of a retail store in order to map those positions to semantic areas like shelves or departments. Unfortunately, as shown in Fig. 3, this is an ill-posed problem: The same pixel may show the projection of objects located at an infinite number of different locations. Through camera calibration, a ray $\vec{r}$ of possible locations (Fig. 3) can be derived but the distance of the object along this ray cannot be determined without additional information or assumptions. Solving this problem is referred to as depth estimation or un-projection and can be achieved by using images from multiple views [48] or additional cues. For example, the relation between image sharpness and the distance of objects to the focal plane can be exploited [49] to this end.

\section{Extension to Multiple Cameras}

So far, we have considered the problem of tracking people using a single camera, but to track people within a larger area more than one camera is necessary. As a consequence, the tracking method has to be adapted. A naïve approach to solve this situation is to stitch video streams from all cameras first and to then perform a monocular tracking on the stitched video as previously discussed. However, this is only viable for a small number of cameras with a very similar orientation.

Typically, more complex solutions are required. As the solution to this problem is mostly independent from the particular use case and the specific implementation of the tracking steps, we are only briefly mentioning two frequent solutions: One are Bayesian belief networks, which can be used merge tracked objects from different cameras [50]. Another one is to map detections to a common three-dimensional coordinate system first before performing the matching which essentially swaps the order of matching and position estimation [51].

\section{LeVeraging Computer Vision based ACQuisition FOR RETAIL RECOMMENDATIONS}

We will now describe a prototypical tracking system for offline retail stores. Its purpose is to generate movement tracks over time for individual customers, from the time the supermarket is entered until it is left again. The tracks are defined with respect to the floor plane of the store which allows to easily map the raw location-based tracks to semantic information such as the visited departments or shelves.

After highlighting the key properties of this specific problem setting from different perspectives in Sec. IV-A, we outline the high-level architecture of the system (Sec. IV-B) and finally identify appropriate building blocks for the individual components of the core tracking algorithm in Sec. IV-C.

\section{A. Offline Retail as a Setting for Visual Tracking}

Several aspects have to be taken into account before designing a visual acquisition system for brick and mortar stores. Some result from the type of acquired data while others concern the visual nature of offline stores from a Computer Vision point of view.

\section{1) Data Perspective:}

a) Validity of Data: The immediate output of tracking, raw sequences of coordinates corresponding to the position of different customers over time, is of little use to a store manager: It has to be enriched by combining it with semantic information about encountered products, shelves or departments. As the mapping between position and semantic information may change over time, for example when products or sections are relocated or even the general layout of a store is modified, special care has to be taken to make sure the semantic data used for different applications is (still) valid.

b) Demographics: Not all customers are alike. Gathering more information about individual customers than just their movements in the store may allow for more precise subsequent analyses and predictions. For example, adults could be differentiated from children in order to see whether and how their shopping habits differ.

c) Privacy: Depending on the country in which a tracking system is used, there may be more or less rigid legal conditions for collecting and storing information. Whereas this is less of a problem for approaches requiring active participation 
of the customer, e.g., downloading a mobile application and agreeing to its terms of service in the case of smartphone-based acquisiton (Sec. II-B), a key property of visual tracking is its reduced saliency to the customer. Regardless of the specific laws applying in practice, the system design therefore needs to aim at finding a balance between a minimum of acquired data and a maximum potential for resulting applications.

d) Ethics: Finally, the idea of analyzing people's behavior to eventually draw profit from it raises ethical questions. As this is a general topic not tied to a particular form of data acquisition and also applies to online retailers, where detailed analyses of individual customers are already common practice, we will not perform a detailed evaluation in this paper.

2) Computer Vision Perspective: From a Computer Vision perspective, offline retails such as supermarkets, hardware stores or even malls pose an interesting environment for videobased tracking. They come with both challenges but also opportunities for simplifying assumptions.

a) Challenges: The main challenge lies in the sheer size of the area in which people have to be tracked. Even for comparatively small stores, multiple cameras are necessary to cover every spot where customers may move, making it necessary to join tracking information from multiple cameras. Also, the temporal extent of tracking is significant. The time spent in a larger store can amount from many minutes to a few hours during which a person has to be followed reliably. Temporary occlusions can occur when a person is hidden from a camera's sight by a stationary object or another person. Similarly, the large number of different objects in various shapes and colors found in a typical store offers potential for false positive detections. Position estimation (Sec. III-C) is complicated by the typically small spatial overlap between video streams from different cameras: From an economical point-of-view, it is desirable to use as few cameras as possible with as little overlap as possible to save hardware and energy costs, which is diametrically opposed to the requirements for position estimation. Finally, the comparatively unusual view on tracked people, often from high above, makes it difficult to compile suitable datasets of similar views to train apperancebased detection methods.

b) Pleasant Properties: Regardless of the preceding challenges, many pleasant properties of offline retail environments go a long way towards making visual tracking a good solution for data acquisition. First, for reasons of product presentation, stores are typical lit brightly with few shadows which guarantees video data of high contrast. There are virtually no illumination fluctuations in space and over time and therefore only limited appearance changes of tracked objects which simplifies their re-identification. Second, surveillance cameras have a fixed location. As geometric conditions can be calibrated in advance, the estimation of positions (Sec. III-C) becomes easier. Third, the store environment, too, is largely static resulting in a consistent background that can be modeled well and allows to detect actually moving objects in the foreground. Lastly, we are interested in tracking objects of a particular type which usually do not move very fast; this means we can detect them based on their look and spatial proximity can serve as an important cue in matching.

\section{B. Overview of the System}

The structure of our proposed system is depicted in Fig. 1. On the hardware side (Fig. 1, left), a network of IP cameras provides the input for our data acquisition. As such camera systems today are the state-of-the-art for video surveillance we can assume a high availability in shops and supermarkets. The tracking algorithm itself is running on a workstation or server equipped with one or multiple GPUs. Such powerful hardware is necessary to provide real-time results in the tracking. Additionally, this server or workstation is where the real-time track data is maintained. In this way, constantly updating it based on the tracking algorithm is more feasible. To store and query offline data, i.e., completed tracks of customers who have already left the retail, a data warehouse is used which can run on the same physical hardware as the tracking method or on a separate server. While the tracking algorithm is continuously performing cycles of detection, tracking and position estimation, the data warehouse provides an API which offers access to completed movement paths as well as the tracks of customers currently shopping. To ensure data validity, the API does not only offer to query raw tracking coordinates but also semantically enriched tracks, e.g., sequences of visited departments in the market. Several applications (Fig. 1, right) are fed by data queried via the API as well as by intermediate analyses (Fig. 1, middle). Queries for offline data can be handled by the warehouse itself whereas requests for online data are forwarded to the computation workstation. When a movement track is finalized, i.e., the tracked person leaves the store, it is pushed to the data warehouse for permanent storage. To ensure maximal conformance with data protection laws, only the raw location data but no further information is stored, e.g., any data on a customer's appearance which has been used for tracking is discarded. This avoids that the stored data can be considered "personal data" (data which is tied to a particular, identifiable person) and circumvents resulting legal issues.

\section{Optimal Algorithmic Building Blocks for Tracking}

Taking into account the previously identified peculiarities of the problem we want to solve (Sec. IV-A2) we can identify appropriate building blocks that maximize the robustness of data acquisition.

1) Detecting People: The large spatial extent of retail stores can typically only be covered using multiple cameras. Additionally, high resolution video data is required to detect people, even if at a distance. This results in large amounts of data which have to be processed for the detection, making a particularly fast detection necessary. A second factor influencing the choice of detection method is the largely static background (Sec. IV-A2) which can and should be utilized in the detection.

We propose a combination of a real-time appearance-based detection method with an additional background modeling 
approach to incorporate temporal cues. For the former, a stateof-the-art method such as YOLO [40], which is based on Deep Learning, has shown the ability to detect objects at rates of around 50 frames per second. Thus, even for a large number of cameras a high-frequent detection is possible. Even if pretrained versions of YOLO detecting multiple object classes exist it is preferable to fine-tune the method to optimize accuracy. Here, focusing on the detection of human heads instead of full people can prove to be more flexible in practice: While the shape and appearance of human bodies varies strongly from different perspectives, due to their spherical shape, heads show less variation. Consequently, a method detecting heads behaves more robustly for a wider range of possible camera positions. The per-frame appearance-based detection can be accompanied by a background modeling approach to distinguish foreground objects from the mostly static background. Due to the beneficial conditions discussed in Sec. IV-A2 simple but efficient and well tested methods such as the one by Stauffer et al. [42] are sufficient. The two detection methods then can be combined, e.g., by using YOLO with a low detection threshold, i.e., producing potentially several false positive detections, and discarding those which are located in background areas.

2) Tracking: As discussed in Sec. III-B, matching detections is an assignment problem which we can solve using wellestablished methods such as the Kuhn-Munkres algorithm [45]. The key to success is a suitable combination of multiple cues in order to form a robust distance measure between detections. A first important factor is spatial proximity. As people in a store typically only move at walking speed and the identified detection methods (Sec. IV-C1) provide several detections per second, we can expect detections corresponding to the same person to be spatially close for subsequent frames. A way to incorporate this is to compute the overlap of bounding boxes between all pairs of detections. The larger the overlap, the smaller the distance for matching. However, in a crowded store, spatial proximity cannot be used as the only cue. An additional cost term based on appearance is necessary to distinguish customers close to each other. A popular and fast-to-compute choice are HOG descriptors [35]. The resulting distance term for these descriptors can be based on standard mathematical norms such as the $L^{1}$-norm.

3) Determining Position: In general, we cannot expect that each point in a retail store will be visible in more than one video stream, which is a pre-requisite for many position estimation approaches. To map positions in the two-dimensional camera image to locations in the store (Fig. 2, right) we therefore have to use heuristic assumptions. Fortunately, our tracking is performed in a static environment that is known in advance We propose the algorithm depicted in Fig. 3 which is adapted from [51]: Assuming an average height, e.g., $175 \mathrm{~cm}$, for customers moving in the store, we can model a virtual plane that detected heads are expected to move in. The position of the person corresponding to a detected head is then derived by intersecting the camera's view ray with the virtual plane and projecting it to the shop floor. In geometrical terms, assuming a previously calibrated camera, if a head is detected in the

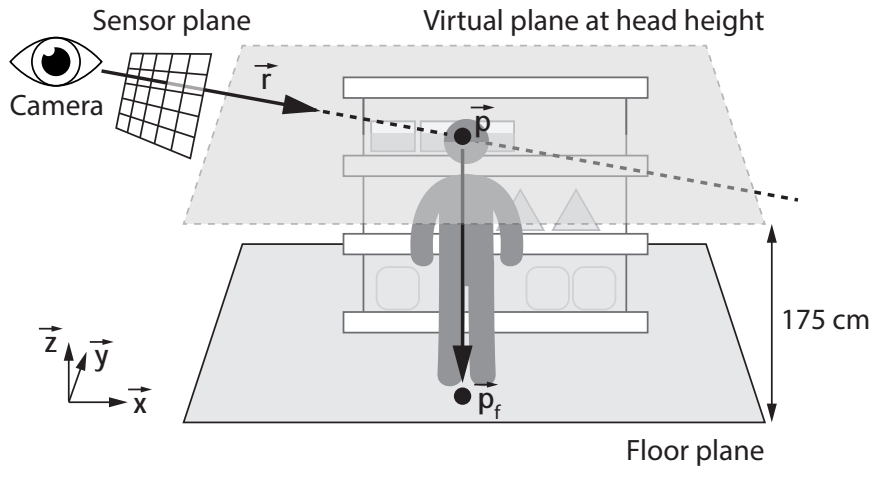

Fig. 3. Employing heuristics and calibration of the camera system used, customer locations can be estimated using straightforward geometrical computations, even if just a single view is available.

camera image, we know the camera ray $\vec{r}$ in three-dimensional space corresponding to the central pixel of the detection. The camera can then be intersected with a virtual plane, parallel to the floor plane, located at the average head height. This yields a point $\vec{p}$, an estimate for the center of the person's head in three-dimensional space. Finally, projecting $\vec{p}$ to the floor plane along $-\vec{z}$ yields $\vec{p}_{\mathrm{f}}$, i.e., the point on the shop floor where the person is standing.

The approach works best if the angle between the negative floor normal $(-\vec{z}$ in Fig. 3$)$ and the camera ray is small. If both are parallel, i.e., the camera is placed on the ceiling, facing downwards, the estimated position is correct; if the angle becomes larger, the estimate becomes worse if a customer's height deviates more from the assumed average height. The estimated position can also be post-processed to avoid invalid data. For example, locations which are known to not be accessible because they are covered by shelves or other objects can be mapped to the closest valid position.

\section{Establishing ReAl-time Applications}

Having introduced a real-time visual tracking system (Sec. IV) which can provide movement paths of customers in a store and their sequences of visited locations (shelves, i.e., product categories, or departments), we can now outline a number of (real-time) applications enabled by this type of data. For each application, we will discuss its benefits and draw connections to similar approaches taken by online retailers.

\section{A. Real-time Recommendations}

In Sec. II-A we identified real-time recommendations as one of the main competitive advantages of online retailers. Using our acquisition system, we can take a similar approach for offline retailers. Sequences of visited shelves and product categories from completed shopping sessions serve as training data for supervised learning methods which then can predict the sections in a store that are likely visited next by customers. For example, Liu et al. [52] proposed an approach for spatial predictions such as that of the next location visited by a traveller or of the city likely attacked next by terror organizations, which could be adapted to this end. Based on this, recommendations, 
e.g., of premium products located in the respective sections, can be targeted at them via large displays distributed throughout the store when a customer is passing by them (cf. Fig. 1). For example, someone who has previously visited shelves containing barbecue products can be exposed to advertisements for premium craft beer while (most likely) on the way to the beverage department.

Currently, brick and mortar stores have to resort to static flyers or in-store advertisement boards to emphasize a small selection of products. The application described can improve upon this situation by finally enabling personalized ads and recommendations in a similar style as in online retail.

A few shortcomings remain: While online retailers can leverage detailed user profiles taking into account individual previous purchases, the proposed recommender system is based only on information from the current shopping session as personal data cannot be stored permanently for data protection reasons (Sec. IV-A). In this scenario, specialized session-based and sequence-aware recommenders may prove useful [53]. Also, the limited advertisement space provided by the displays has to be shared among all customers in a store. The latter problem can be addressed by prioritizing target customers based on their proximity to a display or the confidence of the prediction.

\section{B. Management Interface}

The location-based data we can acquire via our system also provides useful insights to store managers. Both logs of completed shopping sessions and current movement tracks can be aggregated in a multitude of ways to yield real-time dashboards showing information such as the current number of customers in the checkout area or long-term reports on the popularity of individual departments or even shelves. Such reports can help a store manager to check if the actual behavior and usage of a shop coincides with his or her intentions and to take corresponding decisions.

While extremely useful for decision makers, similar reports can up to now only be acquired by costly and laborious manual studies that only capture the situation at a single point in time. The visual tracking system we propose delivers the same information at no additional costs, on a permanent basis and without temporal delays. In this way, the current information gap to online retail, where similar data can already be acquired effortlessly, can be reduced to a minimum.

\section{Efficient Staff Deployment}

A common situation experienced by customers in physical retail is that of being offered support by staff even when not needed or desired which can cause annoyance. At the same time, there are situations when customers would like personal support but no staff seems to be available. Both problems could be resolved by an automated recognition of customers looking for help or advice, regarding such situations as anomalies in customer behavior and detecting them among real-time movement paths by means of unsupervised learning methods. A sequential anomaly detection could be addressed either on a spatial level, e.g., using methods previously developed for surveillance scenarios [54], or from a semantical point of view, considering the sequence of market locations or section visited by a person. In the latter case, similarities to anomaly detection in (business) processes exist, for which solutions like trace clustering [55] are available.

Today, retail stores require well-trained staff that is able to recognize exactly when to offer support to customers without risking to bother the latter. However, even for experienced shop assistants, it is impossible to keep an eye on all customers in a large store at the same time. Automated anomaly detection might be a viable solution.

Drawing a connection to online stores, a few online shops have tried to address the issue by simple heuristics: If a customer spends too much time in a particular step of the shopping process, a chatbox to talk to a bot or a real salesperson pops up. While this can help to mitigate a customer's frustration, virtual contact cannot replace interaction with a real human as it is possible in a physical store. The human factor is one of the few advantages of brick and mortar businesses and with the assistance of an automated detection systems, the latter could use this opportunity to play to their strengths better.

\section{Shoplifting Prevention}

Real-time tracking can also aid the identification of behaviour associated with shoplifting attempts. A recent study [13] estimated the yearly volume of "shrink" due to shoplifting at more than 100 billion dollars. The state of the art in mitigating the problem is to use different forms of tags, which can however be removed by a thief in many cases, alongside the deployment of staff members or specialized detectives.

Similar to the detection of customers requiring assistance (Sec. V-C), the issue can be addressed by a form of sequential anomaly detection with respect to customer behavior. This could help to save costs in two ways. First, costs of hiring additional staff or detectives who still cannot observe all customers in a store at the same time. And second, of course, costs due to actual cases of shoplifting. This could close the gap to e-commerce where the problem of shoplifting does not exist in the same way.

\section{E. Shop Layout Optimization}

Currently, the layout of a physical store is determined by following one of many approaches. Products can be organized according to their expiration properties, their type, or even proven consumption patterns. Many of these aspects are universal and do not depend on a specific store environment but the way a shop is designed and organized influences shopping behavior to a large extent [56]. Therefore, it is important for shop owners to have data which to base their decisions regarding shop layout on. Our acquisition system could provide such data on a permanent and up-to-date basis. Even more, the effects of layout changes could be tracked immediately and thoroughly computing statistics of interest at regular intervals and, when combined with machine learning based predictions, maybe even enable semi-automatic layout optimization that is able to predict how a particular change would influence 
shopping behavior. Inspiration can be taken from the related problem of facility layout optimization [57] which has been addressed much more thoroughly in research than (automated) retail-specific optimization [58]. In this context, often, genetic algorithms [59] have been the method of choice due to their applicability even for complex optimization problems which are impossible to tackle analytically.

\section{CONCLUSION}

In this paper, we have bridged the gap between offline and online retail by proposing a conceptual visual data acquisition system. The location-based data it acquires results in valuable applications for physical retailers that can be used to gain insights into customer behavior and to create services that enhance both the customer's experience and the business' revenue. In the design of our method, we were driven by the shortcomings of existing data acquisition methods: additional hardware requirements, spatial imprecision and, most of all, a need of significant participation by customers. While we have discussed how to overcome these flaws, an extensive case study is necessary to validate our approach and to identify ways to further improve it.

Beyond these technical challenges, another topic requiring further evaluation are potential risks for both retailers and customers. Many customers fear potential data breaches and resulting abuse of the personal data [60]. This in turn becomes a problem for retailers as they may lose those customers to their competitors and, in order to prevent data breaches, have to take expensive countermeasures to ensure data protection. A recent example of that is the German retailer Real which had to cancel a test in which customers' facial expressions in front of advertisement displays were analyzed using cameras [61]. Despite these issues, a recent report by McKinsey concludes that companies that use customer data in general outperform their competitors by $85 \%$ when it comes to sales growth and more than $25 \%$ in gross margins [60], indicating that the benefits of data analysis outweigh their risks.

Another major topic for future work revolves around the question of whether, how, and to which extent to integrate personal data: Online retailers can leverage detailed user profiles, taking into account individual previous purchases and behavior. A similar approach has been taken by physical retail in the form of loyalty programs. Trying to combine such information with tracking data would pose technical and, even more so, legal challenges but also offers potential for more personalized recommendations and detailed analyses and could pave the way to integrating our system into the omni-channel retail landscape. A compromise in this regard could be to refrain from the processing of personal data which can be tied to a particular person but to integrate demographic data which could likely be acquired by a visual acquisition, too.

Despite these limitations, the analysis of anonymous movement paths as proposed in this paper can already be used for several interesting applications (Sec. V) that provide clear benefits for shop owners and help narrowing the current gap to online retailers.

\section{ACKNOWLEDGMENT}

This research is a part of the European Training Network project PERFORM that has received funding from the European Unions Horizon 2020 research and innovation programme under the Marie Skodowska-Curie grant agreement No 765395. This research reflects only the authors' view, the European Commission is not responsible for any use that may be made of the information it contains.

Furthermore, part of this work is based on Visual In-store Customer Analytics and Recommendations (VICAR), a project partly funded by the German ministry of education and research (BMBF), reference number 01S17085C. Again, the authors are responsible for the publications content.

In our figures, we used the following, publicly available icon graphics: Hub, Focus, Dashboard, Recommendation, Alarm, Salesman and Person icon by Freepik. PC icon by prettycons. Server and Graph icon by Smashicons. Track icon by Good Ware. CCTV icon by Those Icons. Neural network icon by Trevor Dsouza.

\section{REFERENCES}

[1] I. Statista. (2019) Total retail sales worldwide from 2015 to 2020 (in trillion u.s. dollars). [Online]. Available: https://www.statista.com/statistics/443522/global-retail-sales/

[2] D. T. T. Limited. (2018) Global powers of retailing 2018. [Online]. Available: https://www2.deloitte.com/ content/dam/Deloitte/at /Documents/about-deloitte/ global-powers-of -retailing-2018.pdf

[3] I. Statista. (2019) Retail e-commerce sales worldwide from 2014 to 2021 (in billion u.s. dollars). [Online]. Available: https://www.statista.com/statistics/379046/worldwide-retail-ecommerce-sales/

[4] B. Berman, "Flatlined: Combatting the death of retail stores," Business Horizons, vol. 62, no. 1, pp. 75-82, 2019.

[5] J. Wang and Y. Zhang, "Opportunity model for e-commerce recommendation: right product; right time," in Proceedings of the 36th international ACM SIGIR conference on Research and development in information retrieval. ACM, 2013, pp. 303-312.

[6] D. Jannach and G. Adomavicius, "Price and profit awareness in recommender systems," arXiv preprint arXiv:1707.08029, 2017.

[7] G. Linden, B. Smith, and J. York, "Amazon. com recommendations: Item-to-item collaborative filtering," IEEE Internet computing, no. 1, pp. $76-80,2003$.

[8] D. Rigby, "The future of shopping," Harvard business review, vol. 89, no. 12, pp. 65-76, 2011.

[9] G. Kalyanaram and Z. Z. Aung, "Value-added ecosystem and customer experience enhancement framework," Kindai Management Review, vol. 3, pp. 49-62, 2015.

[10] M. Bourlakis, S. Papagiannidis, and F. Li, "Retail spatial evolution: paving the way from traditional to metaverse retailing," Electronic Commerce Research, vol. 9, no. 1-2, pp. 135-148, 2009.

[11] E. T. Bradlow, M. Gangwar, P. Kopalle, and S. Voleti, "The role of big data and predictive analytics in retailing," Journal of Retailing, vol. 93, no. 1, pp. 79-95, 2017.

[12] W. Zhang, T. Matsumoto, J. Liu, M. Chu, and B. Begole, "An intelligent fitting room using multi-camera perception," in Proceedings of the 13th international conference on Intelligent user interfaces. ACM, 2008, pp. 60-69.

[13] CNBC. (2013) Shoplifting cost the world \$ 112 billion last year [Online]. Available: https://www.cnbc.com/2013/11/13/retail-theft-costthe-world-112-billion-last-year.html

[14] A. Asthana, M. Crauatts, and P. Krzyzanowski, "An indoor wireless system for personalized shopping assistance," in Mobile Computing Systems and Applications, 1994. WMCSA 1994. First Workshop on. IEEE, 1994, pp. 69-74.

[15] B. Fang, S. Liao, K. Xu, H. Cheng, C. Zhu, and H. Chen, "A novel mobile recommender system for indoor shopping," Expert Systems with Applications, vol. 39, no. 15, pp. 11 992-12000, 2012. 
[16] Z. Zheng, Y. Chen, S. Chen, L. Sun, and D. Chen, "Location-aware poi recommendation for indoor space by exploiting wifi logs," Mobile Information Systems, vol. 2017, 2017.

[17] Y. Chen, Z. Zheng, S. Chen, L. Sun, and D. Chen, "Mining customer preference in physical stores from interaction behavior," IEEE Access, vol. 5, pp. 17 436-17449, 2017.

[18] K. Finkenzeller, RFID handbook: fundamentals and applications in contactless smart cards, radio frequency identification and near-field communication. John Wiley \& Sons, 2010.

[19] F. Caro and R. Sadr, "The internet of things (iot) in retail: Bridging supply and demand," Business Horizons, vol. 62, no. 1, pp. 47-54, 2019

[20] W. T. So and K. Yada, "A framework of recommendation system based on in-store behavior," in Proceedings of the 4th Multidisciplinary International Social Networks Conference on ZZZ. ACM, 2017, p. 33

[21] D. V. d. S. Silva, R. d. S. Silva, and F. A. Durão, "Recstore: Recommending stores for shopping mall customers," in Proceedings of the 23rd Brazillian Symposium on Multimedia and the Web. ACM, 2017, pp. 117-124.

[22] S. S. Chawathe, "Beacon placement for indoor localization using bluetooth," in 2008 11th International IEEE Conference on Intelligent Transportation Systems. IEEE, 2008, pp. 980-985.

[23] E. Lacic, D. Kowald, M. Traub, G. Luzhnica, J. Simon, and E. Lex "Tackling cold-start users in recommender systems with indoor positioning systems." in RecSys Posters, 2015.

[24] P. Christodoulou, K. Christodoulou, and A. S. Andreou, "A real-time targeted recommender system for supermarkets," in 9th International Conference on Enterprise Information Systems. SciTePress, 2017.

[25] A. J. Newman, K. Daniel, and D. P. Oulton, "New insights into retail space and format planning from customer-tracking data," Journal of Retailing and Consumer Services, vol. 9, no. 5, pp. 253-258, 2002.

[26] A. W. Senior, L. Brown, A. Hampapur, C.-F. Shu, Y. Zhai, R. S. Feris, Y.-L. Tian, S. Borger, and C. Carlson, "Video analytics for retail," in Advanced Video and Signal Based Surveillance, 2007. AVSS 2007. IEEE Conference on. IEEE, 2007, pp. 423-428.

[27] E. Frontoni, P. Raspa, A. Mancini, P. Zingaretti, and V. Placidi, "Customers activity recognition in intelligent retail environments," in International Conference on Image Analysis and Processing. Springer, 2013, pp. 509-516.

[28] J. Liu, Y. Gu, and S. Kamijo, "Customer behavior recognition in retail store from surveillance camera," in 2015 IEEE International Symposium on Multimedia (ISM). IEEE, 2015, pp. 154-159.

[29] A. T. Duchowski, "Eye tracking methodology," Theory and practice, vol. 328 , p. 614, 2007

[30] P. T. Huddleston, B. K. Behe, C. Driesener, and S. Minahan, "Insideoutside: Using eye-tracking to investigate search-choice processes in the retail environment," Journal of Retailing and Consumer Services, vol. 43 pp. 85-93, 2018.

[31] M. Sturari, D. Liciotti, R. Pierdicca, E. Frontoni, A. Mancini, M. Contigiani, and P. Zingaretti, "Robust and affordable retail customer profiling by vision and radio beacon sensor fusion," Pattern Recognition Letters, vol. 81, pp. 30-40, 2016

[32] S. Iwase and H. Saito, "Parallel tracking of all soccer players by integrating detected positions in multiple view images," in Pattern Recognition, 2004. ICPR 2004. Proceedings of the 17th International Conference on, vol. 4. IEEE, 2004, pp. 751-754.

[33] O. Sidla, Y. Lypetskyy, N. Brandle, and S. Seer, "Pedestrian detection and tracking for counting applications in crowded situations," in 2006 IEEE International Conference on Video and Signal Based Surveillance. IEEE, 2006, pp. 70-70.

[34] R. Benenson, M. Omran, J. Hosang, and B. Schiele, "Ten years of pedestrian detection, what have we learned?" in European Conference on Computer Vision. Springer, 2014, pp. 613-627.

[35] N. Dalal and B. Triggs, "Histograms of oriented gradients for human detection," in Computer Vision and Pattern Recognition, 2005. CVPR 2005. IEEE Computer Society Conference on, vol. 1. IEEE, 2005, pp 886-893.

[36] P. Viola and M. J. Jones, "Robust real-time face detection," International journal of computer vision, vol. 57, no. 2, pp. 137-154, 2004.

[37] J. A. Suykens and J. Vandewalle, "Least squares support vector machine classifiers," Neural processing letters, vol. 9, no. 3, pp. 293-300, 1999

[38] A. Krizhevsky, I. Sutskever, and G. E. Hinton, "Imagenet classification with deep convolutional neural networks," in Advances in neural information processing systems, 2012, pp. 1097-1105.
[39] A. Vora, "Fchd: A fast and accurate head detector," arXiv preprint arXiv:1809.08766, 2018

[40] J. Redmon, S. Divvala, R. Girshick, and A. Farhadi, "You only look once: Unified, real-time object detection," in Proceedings of the IEEE conference on computer vision and pattern recognition, 2016, pp. 779 788.

[41] M. Abadi, P. Barham, J. Chen, Z. Chen, A. Davis, J. Dean, M. Devin S. Ghemawat, G. Irving, M. Isard et al., "Tensorflow: a system for large-scale machine learning." in OSDI, vol. 16, 2016, pp. 265-283.

[42] C. Stauffer and W. E. L. Grimson, "Adaptive background mixture models for real-time tracking," in cvpr. IEEE, 1999, p. 2246.

[43] A. W. Smeulders, D. M. Chu, R. Cucchiara, S. Calderara, A. Dehghan, and M. Shah, "Visual tracking: An experimental survey," IEEE transactions on pattern analysis and machine intelligence, vol. 36, no. 7, pp. 1442-1468, 2014.

[44] D. Comaniciu, V. Ramesh, and P. Meer, "Real-time tracking of non-rigid objects using mean shift," in Computer Vision and Pattern Recognition, 2000. Proceedings. IEEE Conference on, vol. 2. IEEE, 2000, pp. $142-149$.

[45] H. W. Kuhn, "The hungarian method for the assignment problem," Naval research logistics quarterly, vol. 2, no. 1-2, pp. 83-97, 1955.

[46] D. A. Ross, J. Lim, R.-S. Lin, and M.-H. Yang, "Incremental learning for robust visual tracking," International journal of computer vision, vol. 77, no. 1-3, pp. 125-141, 2008.

[47] C. Dicle, O. I. Camps, and M. Sznaier, "The way they move: Tracking multiple targets with similar appearance," in Proceedings of the IEEE international conference on computer vision, 2013, pp. 2304-2311.

[48] R. Koch, M. Pollefeys, and L. Van Gool, "Multi viewpoint stereo from uncalibrated video sequences," in European conference on computer vision. Springer, 1998, pp. 55-71.

[49] M. Subbarao and G. Surya, "Depth from defocus: a spatial domain approach," International Journal of Computer Vision, vol. 13, no. 3, pp. 271-294, 1994

[50] S. L. Dockstader and A. M. Tekalp, "Multiple camera fusion for multiobject tracking," in Proceedings 2001 IEEE Workshop on Multi-Object Tracking. IEEE, 2001, pp. 95-102.

[51] R. T. Collins, A. J. Lipton, H. Fujiyoshi, and T. Kanade, "Algorithms for cooperative multisensor surveillance," Proceedings of the IEEE, vol. 89 , no. 10, pp. 1456-1477, 2001.

[52] Q. Liu, S. Wu, L. Wang, and T. Tan, "Predicting the next location: A recurrent model with spatial and temporal contexts," in Thirtieth AAAI Conference on Artificial Intelligence, 2016

[53] M. Quadrana, P. Cremonesi, and D. Jannach, "Sequence-aware recommender systems," ACM Computing Surveys (CSUR), vol. 51, no. 4, p. 66, 2018.

[54] R. Laxhammar and G. Falkman, "Online learning and sequential anomaly detection in trajectories," IEEE transactions on pattern analysis and machine intelligence, vol. 36, no. 6, pp. 1158-1173, 2013.

[55] M. Song, C. W. Günther, and W. M. Van der Aalst, "Trace clustering in process mining," in International Conference on Business Process Management. Springer, 2008, pp. 109-120.

[56] P. Singh, N. Katiyar, and G. Verma, "Retail shoppability: the impact of store atmospherics \& store layout on consumer buying patterns,' International journal of scientific \& technology research, vol. 3 , no. 8, pp. 15-23, 2014.

[57] A. Drira, H. Pierreval, and S. Hajri-Gabouj, "Facility layout problems: A survey," Annual reviews in control, vol. 31, no. 2, pp. 255-267, 2007.

[58] T. Ozcan and S. Esnaf, "A discrete constrained optimization using genetic algorithms for a bookstore layout," International Journal of Computational Intelligence Systems, vol. 6, no. 2, pp. 261-278, 2013.

[59] D. E. Goldberg and J. H. Holland, "Genetic algorithms and machine learning," Machine learning, vol. 3, no. 2, pp. 95-99, 1988.

[60] R. W. Palmatier and K. D. Martin, "Understanding and valuing customer data," in The Intelligent Marketers Guide to Data Privacy. Springer, 2019, pp. 133-151.

[61] Focus. (2017) Supermarkt-Kette Real stoppt Gesichtserkennung bei Ladenkameras. [Online]. Available: https://www.focus.de/digita/foto/nachkritik-an-gesichtsanalys-supermarkt-kette -real-stoppt- gesichtserkennungbei-ladenkameras id 7292693.html 